\title{
Viscosity of magmas containing highly deformable bubbles
}

\author{
M. Manga ${ }^{\mathrm{a}, *}$, M. Loewenberg ${ }^{\mathrm{b}}$ \\ ${ }^{a}$ Department of Geological Sciences, University of Oregon, Eugene, OR 97403, USA \\ ${ }^{\mathrm{b}}$ Department of Chemical Engineering, Yale University, New Haven, CT, USA
}

Received 11 October 1999; revised 6 June 2000; accepted 6 June 2000

\begin{abstract}
The shear viscosity of a suspension of deformable bubbles dispersed within a Newtonian fluid is calculated as a function of the shear rate and strain. The relative importance of bubble deformation in the suspension is characterized by the capillary number $(\mathrm{Ca})$, which represents the ratio of viscous and surface tension stresses. For small $\mathrm{Ca}$, bubbles remain nearly spherical, and for sufficiently large strains the viscosity of suspension is greater than that of the suspending fluid, i.e. the relative viscosity is greater than 1. If $\mathrm{Ca}>\mathrm{O}(1)$ the relative viscosity is less than one. In the limit that $\mathrm{Ca} \rightarrow \infty$ (surface tension is dynamically negligible), numerical calculations for a suspension of spherical bubbles agree well with the experimental measurements of Lejeune et al. (1999, Rheology of bubble-bearing magmas. Earth Planet. Sci. Lett., vol. 166, pp. 71-84). In general, bubbles have a modest effect on the relative viscosity, with viscosity changing by less than a factor of about 3 for volume fractions up to 50\%. (C) 2001 Elsevier Science B.V. All rights reserved.
\end{abstract}

Keywords: viscosity of magmas; deformable bubbles; surface tension

\section{Introduction}

The vesiculation of a magma affects its chemical composition and rheological behavior. For example, it is well known that the exsolution of water to form bubbles will increase the viscosity of the melt. The effects of bubbles on the rheology of magmas, however, are not as well characterized. For instance, the experimental measurements of Stein and Spera (1992) suggest that the presence of bubbles may increase the viscosity of the suspension, whereas other experimental measurements suggest that the viscosity may decrease (Bagdassarov and Dingwell, 1992; Lejeune et al., 1999).

The analysis of the mechanical properties of

\footnotetext{
* Corresponding author. Tel.: +1-541-346-5574; fax: +1-541346-4692.

E-mail address: manga@newberry.uoregon.edu (M. Manga).
}

suspensions originated with a study by Einstein (1906). In a dilute suspension, one in which the suspended particles are sufficiently well separated that they do not interact hydrodynamically, the relative viscosity can be written as:

$$
\begin{aligned}
\mu_{\text {rel }} & \equiv \frac{\text { shear viscosity of suspension }}{\text { shear viscosity of the suspending fluid }} \\
& =1+f \phi .
\end{aligned}
$$

Here $\phi$ is the volume fraction of the dispersed phase, and $f$ is a constant that depends on the properties of suspended particles. For rigid spheres, Einstein (1906) found that $f=5 / 2$ if the Reynolds number based on the particle size is small (assumed to be the case for the rest of this paper). For bubbles that remain spherical due to surface tension, Taylor (1932) found that $f=1$, that is, $\mu_{\text {rel }}$ increases by an amount equal to the 
volume fraction of bubbles. At higher volume fractions, interactions between the dispersed particles will affect $\mu_{\text {rel }}$, and Eq. (1) may no longer be a good approximation for $\mu_{\text {rel }}(\phi)$.

In a suspension of bubbles, deformation of the bubbles will also affect $\mu_{\text {rel }}$. Bubble deformation depends on the relative magnitude of shear stresses that act to deform bubbles compared with surface tension stresses that act to keep bubbles spherical. The ratio of these two stresses is usually characterized by a dimensionless number called the capillary number:

$\mathrm{Ca} \equiv \frac{\text { viscous tresses }}{\text { surface tension stresses }}=\frac{\mu \dot{\gamma} a}{\sigma}$.

Here $\dot{\gamma}, \mu, a$ and $\sigma$ are the shear rate, suspending fluid viscosity, bubble radius, and surface tension, respectively. A "bubble" is defined as any void filled with fluid that is much less viscous than the suspending fluid.

Manga et al. (1998) calculated $\mu_{\text {rel }}$ for suspensions of interacting and deforming bubbles in the limit of small bubble deformation $(\mathrm{Ca} \leq 0.3)$. Many magmas, however, have $\mathrm{Ca} \gg 1$ due to their large viscosities (see table 1 in Manga et al., 1998). The purpose of this short communication is to extend the numerical results to the limit that surface tension becomes dynamically negligible. The numerical results agree with recently published experimental data in Lejeune et al. (1999).

\section{Numerical calculations}

The purpose of the numerical calculations is to determine the effects of bubbles on the relationship between the average rate-of-strain and stress tensors, $\bar{G}_{i j}$ and $\bar{T}_{i j}$, respectively. Numerical calculations are performed using the boundary integral numerical techniques described in Loewenberg and Hinch (1996). In the numerical simulations, we apply a given rate of simple shear to the suspension (e.g. $\bar{G}_{12}=\dot{\gamma}$ and the other components of $\bar{G}_{i j}$ are zero) and calculate $\bar{T}_{i j}$. Eqs. (7)-(9) in Manga et al. (1998) show how $\bar{T}_{i j}$ is calculated. The relative viscosity is then given by:

$\mu_{\text {rel }}=\bar{T}_{12} / \mu \bar{G}_{12}$.

We consider two model problems. For the limit of dilute suspensions, we determine $f \mu$ in Eq. (1) by calculating the deformation of a single bubble and the bubble's effect on the stress tensor (Fig. 1a). For larger concentrations we place 8 or 20 deformable bubbles in a triply periodic cell (Fig. 1b). The shape of each bubble is represented numerically by a grid of 320 and 1620 triangles for the suspension and single bubble simulations, respectively. In a dilute suspension, $f=-5 / 3$ for spherical bubbles in the limit of vanishing interfacial tension (Dewey, 1947; Eshelby, 1957). The numerical calculations for a spherical bubble (and $\gamma=0$ ) give -1.646 and -1.662 for bubbles represented by 320 and 1620 triangles, i.e. errors are 1.3 and $0.26 \%$, respectively.

At high $\mathrm{Ca}$ bubbles develop pointed ends, and the numerical difficulty of resolving the high curvature of the bubble surface limits the strains we can compute. Nevertheless, we can still achieve strains of about 1 , and thus larger than the strains often used in experimental rheological measurements (e.g. Bagdassarov and Dingwell, 1992; Lejeune et al., 1999). Such

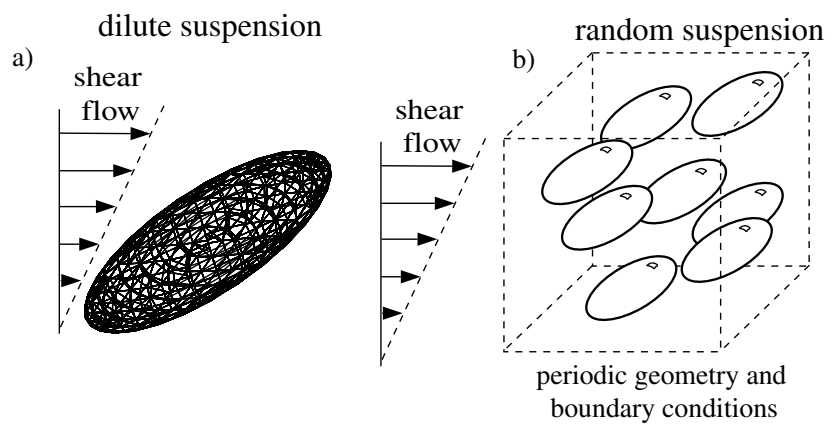

Fig. 1. Geometry of the two problems considered in this study: (a) single bubble; and (b) random suspension. 
strains are also large enough to achieve a quasi steadystate for $\mathrm{Ca}<\mathrm{O}(1)$. The upper value on $\phi(40 \%)$ is governed by the maximum number of initially spherical bubbles of uniform size we can randomly position inside each unit cell.

By mathematical analogy, the effective shear viscosity of suspensions can be equated to the shear modulus of elastic composites in which inclusions are contained within an incompressible matrix. The analogy has limits, however, because the boundary conditions that apply on the surface of bubbles and inclusions will only be the same in the limit of vanishing surface tension. The analogy has some additional limitations if the suspension is not dilute: the effective viscosity depends on the particle distribution, and in a fluid, hydrodynamic interactions between suspended particles affect their spatial distribution. In the discussion that follows, we compare our numerical calculations with various elasticity results, noting that the latter can apply only in the limit that $\mathrm{Ca} \rightarrow \infty$.

\section{Results and discussion}

First we consider dilute suspensions. Fig. 2 shows $f$ in Eq. (1) as a function of shear $(\gamma)$ for various shear rates (proportional to $\mathrm{Ca}$ ). For comparison we show the analytical results for spherical bubbles $(f=1)$ and spherical inclusions (no surface tension: $f=-5 / 3$ ). Because the magnitude of bubble deformation scales with $\mathrm{Ca}, f$ for low $\mathrm{Ca}$ bubbles approaches the spherical-bubble limit. The difference between the spherical-bubble and spherical-inclusion results is due to the different boundary conditions that apply on the bubble surface. The spherical-bubble result of Taylor (1932) is appropriate for small Ca and sufficiently large strains that the deformation has reached a steady value (e.g. see curve for $\mathrm{Ca}=$ 0.1 in Fig. 2). In this case, the component of velocity normal to the bubble surface is zero. In contrast, the inviscid spherical-inclusion result relaxes the zero normal velocity boundary condition and such bubbles would thus be deformed by the flow. In Fig. 2 the bubbles only reach a steady shape, and thus a steady value for $f$, if $\mathrm{Ca} \leq 1$. For larger $\mathrm{Ca}$, once strains are greater than about 1 , we are unable to accurately compute the highly distorted bubble shapes. Nevertheless, the results for $\mathrm{Ca} \gg 1$ are similar to the spherical-inclusion result over the range of strains we have been able to study.

Fig. 2 illustrates two general properties of bubbly suspensions. First, bubbly fluids are shear-thinning, that is, as the shear rate (Ca) increases, $\mu_{\text {rel }}$ decreases. Second, $\mu_{\text {rel }}$ can be greater than 1 if $\mathrm{Ca}$ is sufficiently

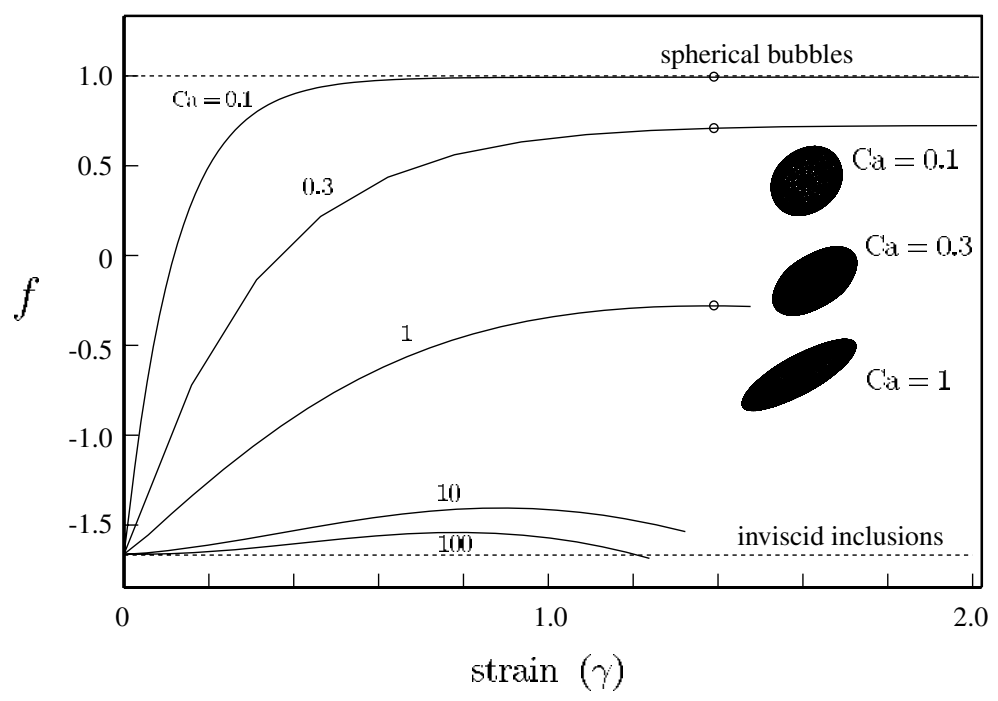

Fig. 2. Relative viscosity ( $f$ defined in Eq. (1)) as a function of strain in the dilute limit (Fig. 1a). Numbers on the curves are the values of Ca. The upper dashed line is the analytical result for a dilute suspension of spherical bubbles; the lower dashed line is the analytical result for a dilute suspension of spherical inclusions (no surface tension). The three bubble shapes are shown for strains of 1.2. 


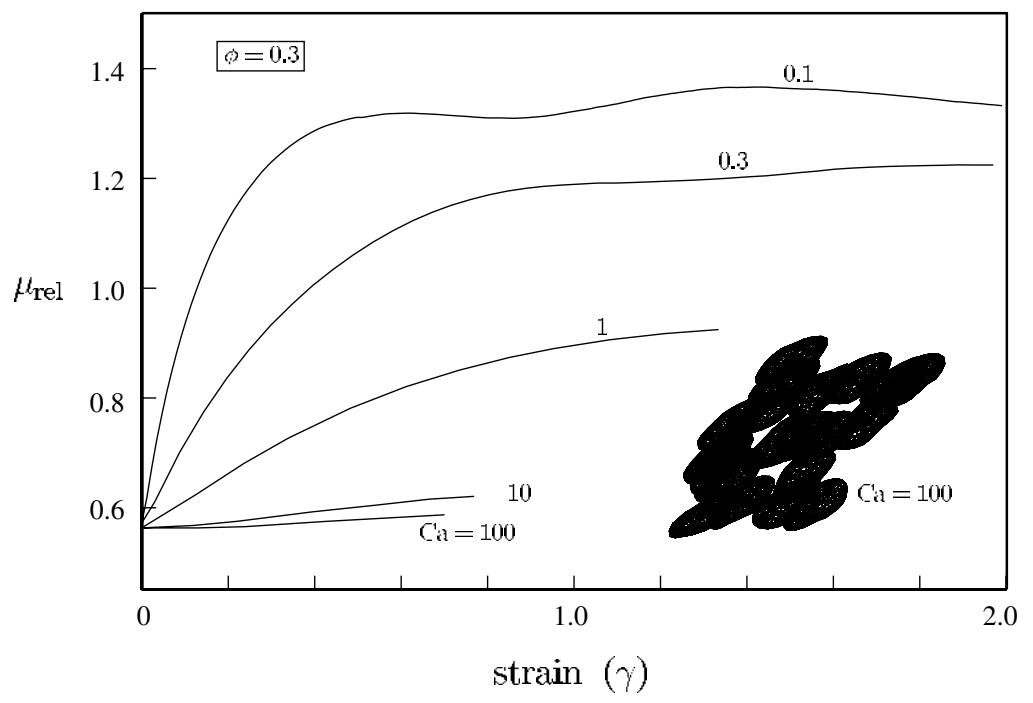

Fig. 3. Relative viscosity as a function of strain for a random suspension (Fig. 1b). Numbers on the curves are the values of Ca. Volume fraction is $30 \%$.

small that bubbles remain nearly spherical. However, $\mu_{\text {rel }}$ can be less than 1 if $\mathrm{Ca}$ is large enough that bubbles can become highly deformed. For reference, examples of bubble shapes are also shown in Fig. 2.

We now consider more concentrated suspensions. Fig. 3 shows $\mu_{\text {rel }}$ as a function of strain for various shear rates. The volume fraction is set to $30 \%$. In the calculations with $\mathrm{Ca}=0.1$ and $\mathrm{Ca}=0.3$ the unit cell in Fig. $1 \mathrm{~b}$ contains 8 bubbles and in the other simulations the unit cell contains 20 bubbles. Again, as $\mathrm{Ca}$ increases, $\mu_{\text {rel }}$ decreases, and $\mu_{\text {rel }}$ can be either $>1$ or $<1$. For $\phi=30 \%, \mu_{\text {rel }}$ increases about $30 \%$ for nearly spherical bubbles $(\mathrm{Ca} \rightarrow 0)$, and decreases about $40 \%$ if surface tension is dynamically negligible $(\mathrm{Ca} \rightarrow$ $\infty)$. Again, numerical limitations prevent the calculations from reaching steady-state if $\mathrm{Ca}$ is greater than about 1 .

Finally, Fig. 4 shows $\mu_{\text {rel }}$ for a suspension of spherical bubbles (infinitesimally small strains) and $\mathrm{Ca} \gg$ 1 (surface tension does not affect $\mu_{\text {rel }}$ ). This limit is analogous to the elasticity problem, as discussed in Section 2. Manga et al. (1998) suggested that in the limit of vanishing surface tension forces $(\mathrm{Ca} \rightarrow \infty)$, $\mu_{\text {rel }}=1-\phi$ if we assume that the bubbles do not affect the flow and viscous dissipation thus only occurs in the liquid phase that occupies a volume fraction of $1-\phi$. This result is analogous to the so- called Voight bound or Voight average which assumes that strain is uniform in the composite (e.g. Watt et al., 1976). In Fig. 4 we show this Voight average, along with the Hashin-Shtrikman upper bound (Hashin and Shtrikman, 1963):

$\mu_{\text {rel }}=1-\frac{5 \phi}{3+2 \phi}$.

The Hashin-Shtrikman lower bound is $\mu_{\text {rel }}=0$. Finally, we include an extrapolation of the dilute limit (Dewey, 1947; Eshelby, 1957). In general, the numerical results for a suspension of randomly positioned spherical bubbles follow the form of the Hashin-Strikman upper bound. Deviations of the numerical results from the extrapolation of the dilute limit are due to the mutual interaction of bubbles.

Fig. 4 also shows the experimental measurements of Lejeune et al. (1999). In these experiments, $\mathrm{Ca} \gg 1$ and the total strains are between 0.05 and 0.40 . Thus a comparison with spherical bubble results in the limit of vanishing surface tension should be a reasonable approximation. Deformation of the bubbles, even in the limit of large Ca will still affect $\mu_{\text {rel }}$ (see curves for $\mathrm{Ca}=100$ in Figs. 2 and 3) though changes are small. In Fig. 4 we have omitted Lejeune et al.'s experimental data obtained at temperatures less than $10^{\circ} \mathrm{C}$ higher than the glass transition temperature because our 


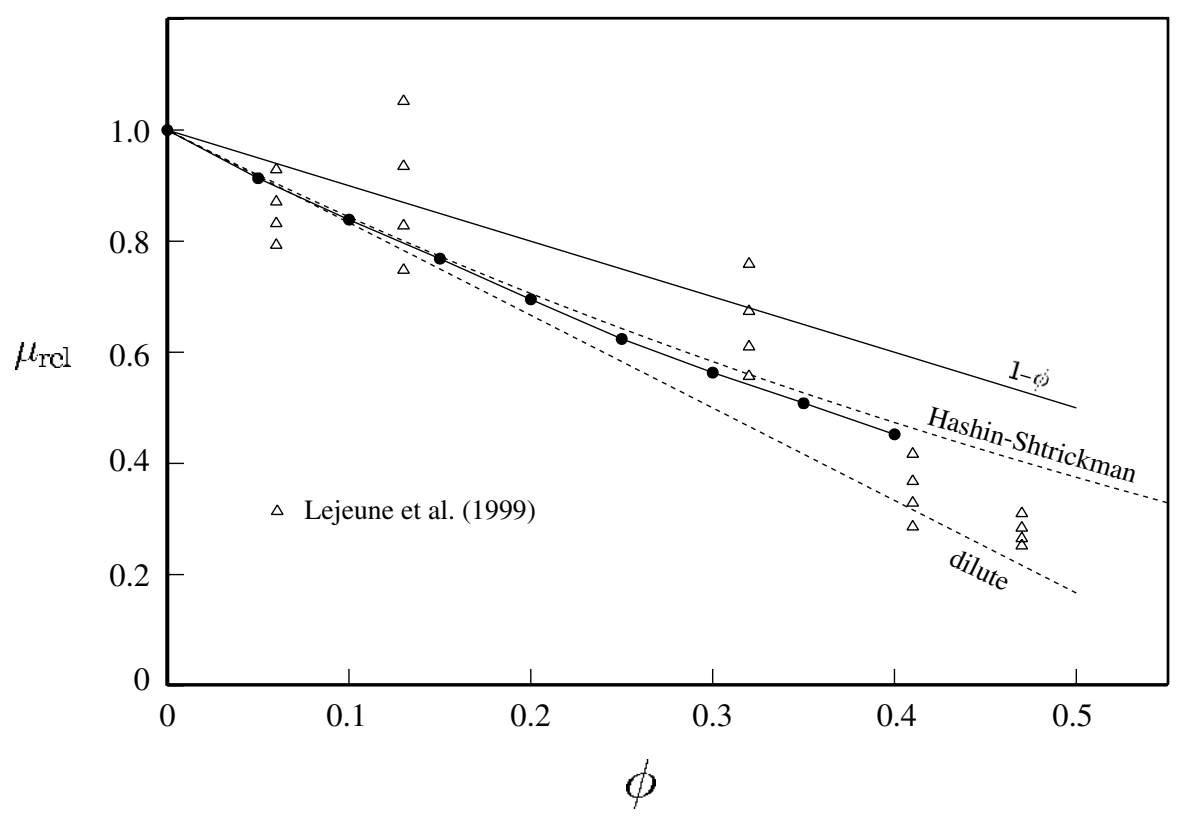

Fig. 4. Relative viscosity for a suspension of spherical bubbles in the limit that $\mathrm{Ca} \rightarrow \infty$ (filled discs). The triangles show the experimental measurements of Lejeune et al. (1999). See Spera and Stein (2000) and Lejeune et al. $(1999,2000)$ for a discussion of the experimental uncertainties. The solid line is the so-called Voight average $(1-\phi)$. The dashed curves are the Hashin-Shtrickman upper bound and an extrapolation of the dilute limit (e.g. Hashin, 1983).

simulations assume that suspending fluid is a Newtonian fluid. Near and below the glass transition temperature, microstructure within the liquid phase may interact with the bubbles and affect $\mu_{\text {rel }}$ (Lejeune et al., 1999). Indeed, the temperature-dependence of $\mu_{\text {rel }}$ in the experimental data suggests this to be the case. Spera and Stein (2000), however, argue that the data of Lejeune et al. (1999) suggest that $\mu_{\text {rel }}$ is independent of temperature and monotonically decreases as $\phi$ increases. In general, Fig. 4 shows that the agreement between the theoretical and experimental results is good.

\section{Concluding remarks}

The relative viscosity of bubbly magmas depends on both the shear rate (i.e. Ca) and the total strain (which affects bubble shape). The theoretical results presented here and in Manga et al. (1998), as well as the experimental results of Lejeune et al. (1999), suggest that bubbles may increase or decrease $\mu_{\text {rel }}$ by a factor of less than about 3 for volume fractions less than 50\%. The magnitude of the effect of bubbles is thus small compared to viscosity changes magmas might experience due to the loss of volatiles, crystallization, or cooling. However, neither the experimental results of Lejeune et al. (1999), nor our numerical results, achieve large enough strains that a quasi-steady state is reached for large $\mathrm{Ca}$.

Although $\mu_{\text {rel }}$ may not change "significantly" due to the presence of bubbles, vesiculation may still have a large effect on magmatic processes. For example, the density difference between magma and crustal rocks (and thus the buoyancy forces driving flow) can change by more than an order of magnitude due to vesiculation of $50 \%$ bubbles. It is also important to recall that $\mu_{\text {rel }}$ describes only one aspect of the rheology of bubbly magmas. In particular, bubbly liquids are shear-thinning (as shown in Figs. 2 and 3), and large normal stress differences are generated by bubble deformation (e.g. Showalter et al., 1978; Stein and Spera, 1992; Manga et al., 1998). The latter may affect the walls of conduits and dikes during the flow of bubbly magmas. 


\section{Acknowledgements}

Work supported by NSF grants CTS-9624615 and EAR-9701768. The authors thank K.V. Cashman and an anonymous reviewer for comments and suggestions.

\section{References}

Bagdassarov, N., Dingwell, D.B., 1992. A rheological investigation of vesicular rhyolite. J. Volcanol. Geotherm. Res. 50, 307-322.

Dewey, J.M., 1947. The elastic constants of materials loaded with non-rigid fillers. J. Appl. Phys. 18, 578-581.

Einstein, A., 1906. Eine neue Bestimmung der Moleküldimensionen, Annal. Physik, 19: 289-306. English translation in Investigation on the Theory of Brownian Motion, Dover, New York, 1956.

Eshelby, J.D., 1957. The determination of the field of an ellipsoidal inclusion and related problems. Proc. R. Soc. London A 241, 376-396.

Hashin, Z., 1983. Analysis of composite materials - A survey. J. Appl. Mech. 50, 481-505.

Hashin, Z., Shtrikman, S., 1963. A variational approach to the elastic behavior of multiphase materials. J. Mech. Phys. Solids 11, 127-140.
Lejeune, A.M., Bottinga, Y., Trull, T.W., Richet, P., 1999. Rheology of bubble-bearing magmas. Earth Planet. Sci. Lett. 166, 7184.

Lejeune, A.M., Bottinga, Y., Trull, T.W., Richet, P., 2000. Rheology of bubble-bearing magmas: Reply to a critical comment by Spera and Stein. Earth Planet. Sci. Lett. 175, 333-334.

Loewenberg, M., Hinch, E.J., 1996. Numerical simulation of a concentrated emulsion in shear flow. J. Fluid Mech. 321, 395-419.

Manga, M., Castro, J., Cashman, K.V., Loewenberg, M., 1998. Rheology of bubble-bearing magmas. J. Volcanol. Geotherm. Res. 87, 15-28.

Schowalter, W.R., Chaffey, C.E., Brenner, H., 1968. Rheological behaviour of a dilute emulsion. J. Colloid Interface Sci. 26, 152-160.

Spera, F.J., Stein, D.J., 2000. Comment on "Rheology of bubblebearing magmas" by Lejeune et al. Earth Planet. Sci. Lett. 175, 327-331.

Stein, D.J., Spera, F.J., 1992. Rheology and microstructure of magmatic emulsions - Theory and experiments. J. Volcanol. Geotherm. Res. 49, 157-174.

Taylor, G.I., 1932. The viscosity of a fluid containing small drops of another fluid. Proc. R. Soc. London A 138, 41-48.

Watt, J.P., Davies, G.F., O'Connell, R.J., 1976. The elastic properties of composite materials. Rev. Geophys. 14, 541-563. 